\title{
Surgicel Nu-Knit hemostat for bleeding control of fragile sternum
}

\author{
Helmut Mair, MD, Ingo Kaczmarek, MD, Martin Oberhoffer, MD, Jan Groetzner, MD, \\ Sabine Daebritz, MD, and Bruno Reichart, MD, Munich, Germany
}

$\mathrm{P}$ rofuse bleeding from the sternal marrow after sternotomy is routinely controlled with bone wax because it is effective, cheap, and therefore favorable. However, bone wax inhibits osseous fusion and promotes infections. ${ }^{1}$ The increasing numbers of patients with high risk for sternal instability and wound-healing complications after cardiac surgery requires improved concepts in bleeding control without the use of bone wax. We present an alternative technique to control bleeding from the sternal bone marrow with patches of oxidized regenerated cellulose (Nu-Knit; Ethicon, Johnson \& Johnson, Somerville, NJ).

\section{Technique}

A 73-year-old woman with unstable angina was referred to our department for urgent surgical revascularization and mitral valve repair. In addition, the patient had insulin-dependent diabetes, chronic obstructive lung disease, and obesity (body mass index, 32.4).

Median sternotomy revealed a fragile sternum and severe osteoporosis with excessive bleeding from the bone marrow. Electrocautery to the periostium was used sparingly to control bleeding, and patches of $3 \times 9$-inch Surgicel Nu-Knit hemostat (one 6 $\times 9$-inch Surgicel Nu-Knit hemostat cut in half) were placed on each side of the sternum (Figure 1). Additionally, towels were wrapped around the absorbable hemostatic patches for their optimal fixation and for atraumatic management of the tissue before the retractor was inserted. After 2 to 3 minutes, a good hemostatic effect with the Surgicel Nu-Knit hemostat (Figure 2) was achieved. Full heparinization (active clotting time $>400$ seconds) was initiated for the extracorporeal circulation. Routine coronary arterial bypass grafting ( 3 grafts) and mitral valve repair were performed. After surgical intervention and decannulation, protamine was administered to antagonize heparin completely. The hemostatic patches were removed from the sternum, and no additional hemostatic agent was administered. Finally, the sternum was closed in a standard fashion with wires. The wound was closed in

\footnotetext{
From the Department of Cardiac Surgery, University of Munich, Munich, Germany.

Received for publication March 24, 2005; accepted for publication April 11, 2005.

Address for reprints: Helmut Mair, MD, Cardiac Surgery, Klinikum Großhadern University of Munich, Marchioninistr. 15, D-81377 Munich, Germany (E-mail: h.mair@hch.med.uni-muenchen.de).

J Thorac Cardiovasc Surg 2005;130:605-6

$0022-5223 / \$ 30.00$

Copyright (C) 2005 by The American Association for Thoracic Surgery doi:10.1016/j.jtcvs.2005.04.013
}

layers, with intracutaneous sutures used for skin closure. The intraoperative and postoperative course of the patient was uneventful. No enhanced bleeding, infection, or sternal wound-healing complication occurred postoperatively.

To date, we have performed this hemostatic procedure in 53 patients at high risk for unstable sternum and infection. We had good or at least satisfactory intraoperative blood control in all cases. We observed no in-hospital mortality. No patient required a reoperation because of bleeding or unstable sternum. None of the patients had a deep sternal wound infection.

\section{Discussion}

Bone wax should not be used excessively and should be avoided in patients at high risk for infection or nonunion altogether. ${ }^{1}$ In particular, bone wax might not even be effective in elderly patients and those with osteoporosis. The spongiosa scaffold of the sternum can be destroyed during setting of bone wax, and the marrow cavity might absorb large quantities of bone wax with still enhanced bleeding. As shown in our case, use of the topical hemostatic agent Surgicel Nu-Knit is a simple, capable, and effective method for sternal bleeding control in a high-risk patient.

Our experience with the conventional Surgicel hemostat for sternal bleeding control has demonstrated safety and practicability, ${ }^{2}$ but it is less efficient than the Surgicel Nu-Knit hemostat. The

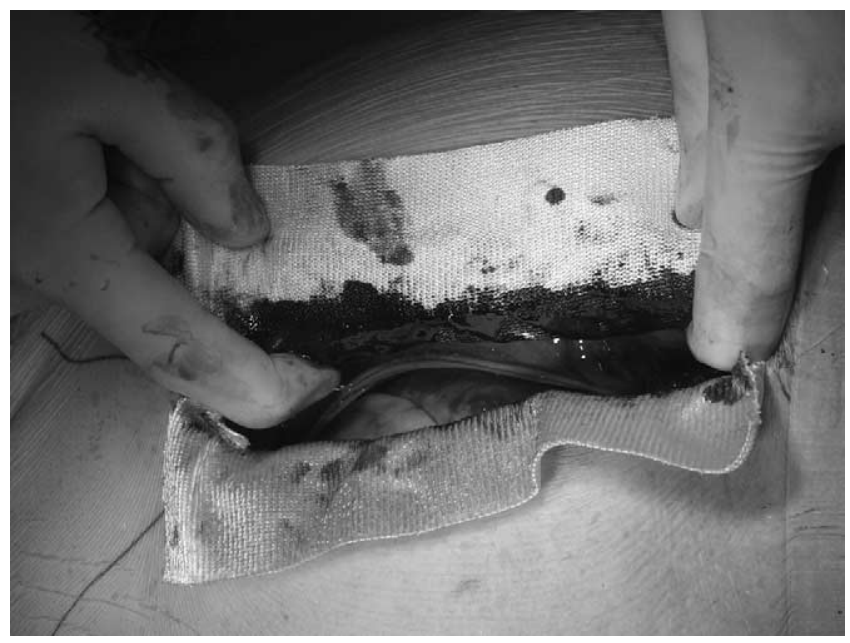

Figure 1. Oxidized regenerated cellulose (Surgicel Nu-Knit hemostat, $3 \times 9$ inches) was placed on both sides of the sternotomy. 


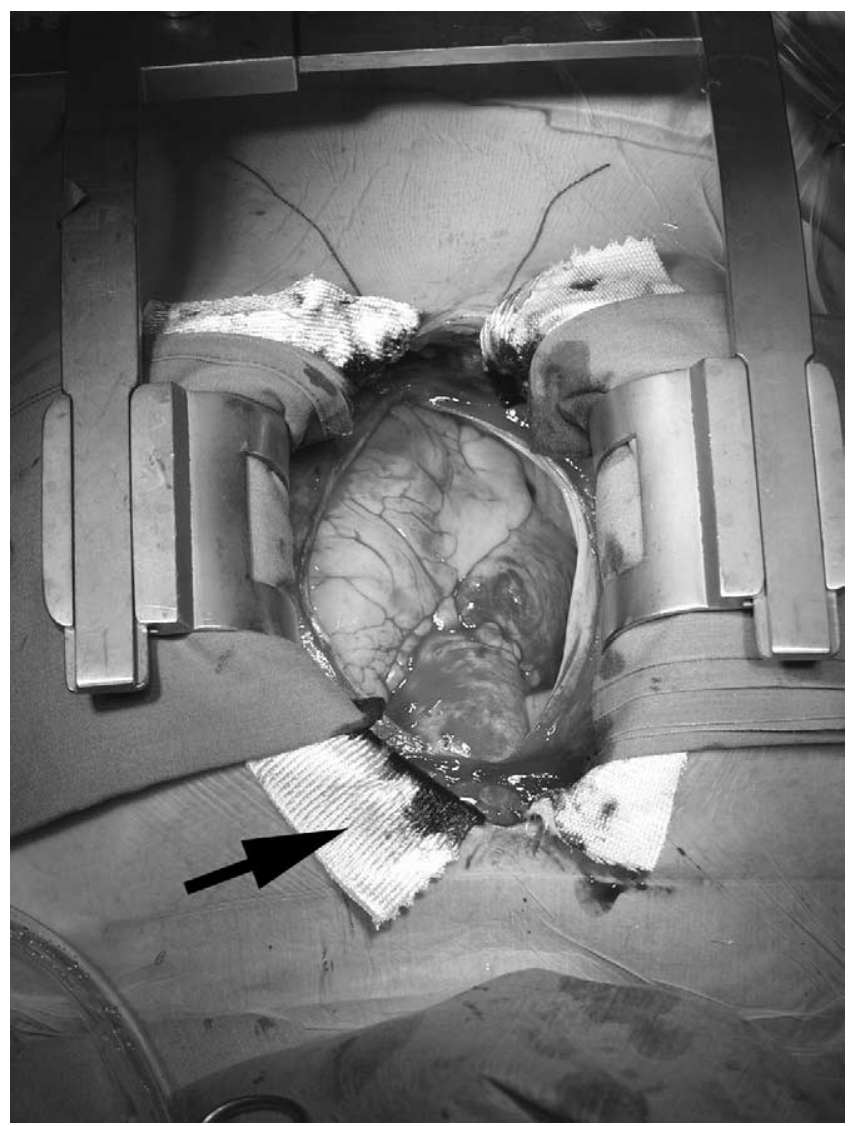

Figure 2. Towels were wrapped around the hemostatic patches (black arrow) for optimized fixation and atraumatic management of the tissue before insertion of the retractor. Full heparinization (active clotting time $>400$ seconds) was initiated. preparation of the oxidized regenerated cellulose as Surgicel $\mathrm{Nu}-$ Knit has triple density, has $150 \%$ thickness, and is more tear proof than the classic Surgicel patch. The enlarged surface matrix provided an additive hemostatic effect as a result of improved platelet adhesion. In addition to the hemostatic effect, oxidized regenerated cellulose has been shown to reduce significantly Staphylococcus aureus growth in vitro, ${ }^{3}$ which represents one of the main bacteria responsible for sternal wound infections. Therefore, a beneficial bactericidal effect in vivo can be presumed. Oxidized regenerated cellulose is a fully resorbable topical hemostatic agent, and usually the Surgicel Nu-Knit patches were removed before sternal wiring. However, accidentally extant material will be reabsorbed in 3 to 5 weeks.

Other agents, like fibrin sealant ${ }^{4}$ or microfibrillar collagen, also have a good hemostatic effect, but they are cost-intensive. In addition, microfibrillar collagen might cause adverse effects because the small diameter of its needle-shaped structure potentially passes the filters of blood-saving devices and enters the patient's circulation. This might result in organ damage (eg, lung or brain)..$^{5}$

In conclusion, oxidized regenerated cellulose patches can be temporarily administered safely and effectively to avoid sternal bleeding complications with consecutive wound-healing problems and mediastinitis.

\section{References}

1. Nelson DR, Buxton TB, Luu QN, Rissing JP. The promotional effect of bone wax on experimental Staphylococcus aureus osteomyelitis. J Thorac Cardiovasc Surg. 1990;99:977-80.

2. Mair H, Schutz A, Lamm P, Reichart B. Control of bleeding from fragile sternum with a resorbable hemostyptic. Ann Thorac Surg. 2001; 71:759-60.

3. Atlas RM, Bartha R. Microbial ecology. 4th ed. Menlo Park (CA): Benjamin/Cummings Science Publishing; 1998. p. 314-5.

4. Pasic M, Hetzer R. Fibrin glue instillation for profuse sternal bleeding. J Thorac Cardiovasc Surg. 2002;124:1247.

5. Robicsek F, Duncan GD, Born GVR, Wilkinson HA, Masters TN, McClure M. Inherent dangers of simultaneous application of microfibrillar collagen hemostat and blood saving devices. $J$ Thorac Cardiovasc Surg. 1986;92:766-70. 